\title{
An Uncommon Cause of Dysarthria in an Elderly Gentleman
}

\author{
Muhammad Noushad $^{1}$ Shakya Bhattacharjee ${ }^{1}$ \\ ${ }^{1}$ Department of Neurology, Plymouth Hospital NHS Trust, \\ Plymouth, United Kingdom
}

J Neurosci Rural Pract 2020;11:357-358

A 76-year-old gentleman presented with mild slurred speech. Five months earlier, he had vomiting and unsteady gait. Magnetic resonance imaging (MRI) head then showed hematoma of the dentate nucleus of the right cerebellum ( $\bullet$ Fig. 1, black arrow). Now the clinical examination revealed mild dysarthria, asymmetric palatal tremor predominantly involving the right palatal arch, and pharyngeal myoclonus ( - Video 1). The current MRI head showed hypertrophy of the con-

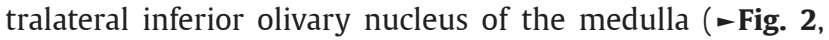

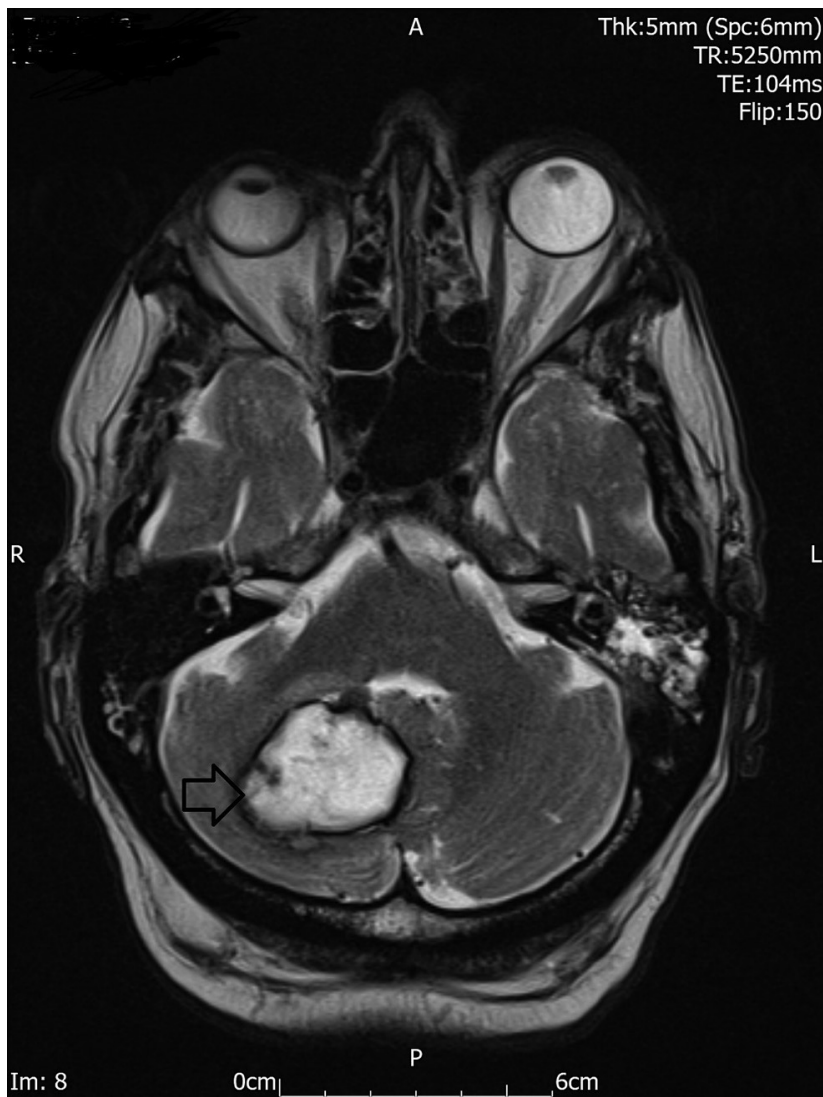

Fig. 1 Axial T2-weighted magnetic resonance imaging (MRI) sequence showing right cerebellar hemorrhage involving the dentate nucleus (black arrow) (dentate nucleus is a part of the Guillain-Mollaret triangle).
Address for correspondence Shakya Bhattacharjee, MRCP (UK), Department of Neurology, Plymouth Hospital NHS Trust, Plymouth PL6 8DH, United Kingdom (e-mail: bubai.shakya@gmail.com).

\section{Video 1}

The video shows asymmetric palatal tremor (right palatal arch > left palatal arch) with intermittent myoclonus of the posterior pharyngeal wall. Online content including videosequences viewable at: https://www.thieme-connect.com/ products/ejournals/10.1055/s-0040-1701552.

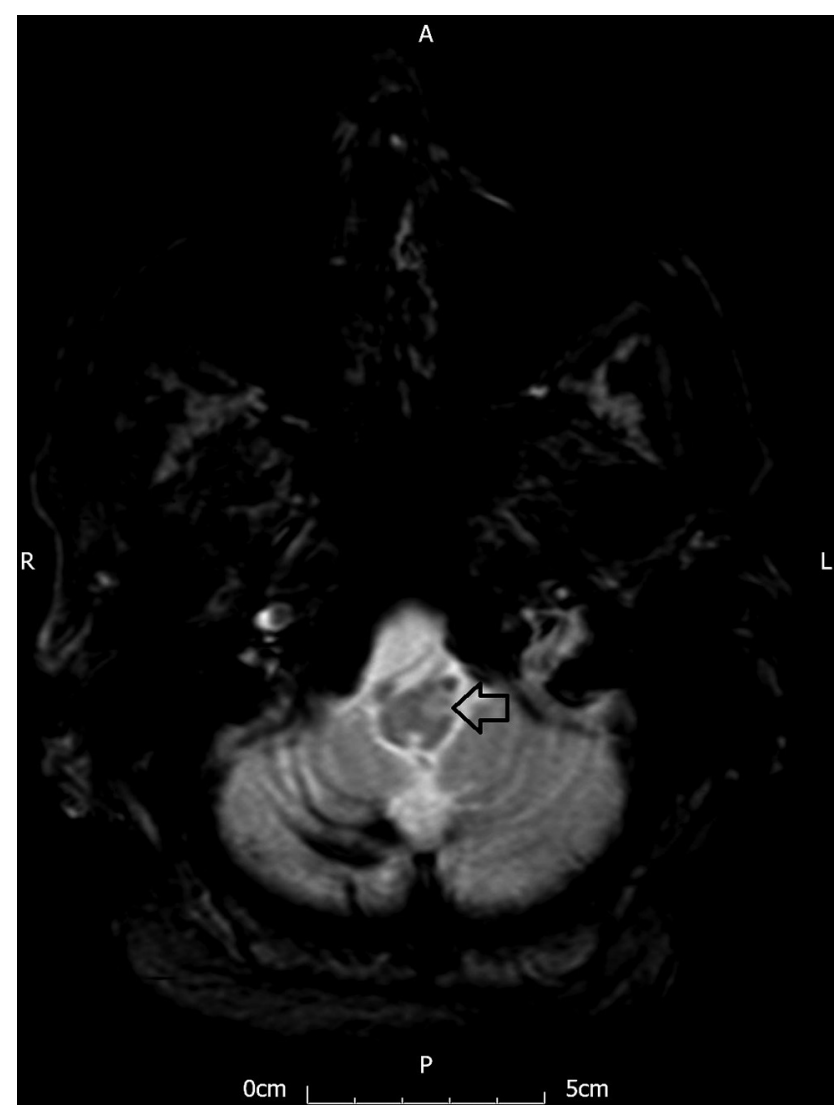

Fig. 2 Axial T1-weighted magnetic resonance imaging (MRI) sequence showing the hypertrophic olivary degeneration of the inferior olivary nucleus of the left hemimedullar (black arrow).
DOI https://doi.org/ $10.1055 / \mathrm{s}-0040-1701552$ ISSN 0976-3147. 
black arrow). He had symptomatic palatal tremor because of the contraction of the levator veli palatine muscle. ${ }^{1}$ We postulate that the pharyngeal myoclonus was due to the common vagal nerve supply. Hypertrophic olivary degeneration occurred secondary to a lesion in the Guillain-Mollaret triangle. ${ }^{2}$ The hallmark clinical feature of a lesion of the GuillainMollaret triangle is the palatal tremor. The unilateral palatal tremor happened due to the hypertrophic degeneration of the contralateral olivary nucleus. ${ }^{3}$ However, though bilateral symmetrical palatal tremor is common but an asymmetric bilateral tremor described in our patient was rarely reported in literature.

The palatal tremor can be essential or idiopathic and symptomatic. The essential palatal tremor occurs due to the contraction of the tensor veli palatine muscle, supplied by the trigeminal nerve. ${ }^{3}$ Often patients complain of an audible ear click. The MR head reveals no abnormalities in a patient with essential palatal tremor. Symptomatic palatal tremor occurs due to the contraction of the levator veli palatine, supplied by the vagus nerve. Many other clinical signs like Holme's tremor, ocular tremor or flutter, or ataxia are found in patients with symptomatic palatal tremor.

\section{Funding}

None.

\section{Conflict of Interest}

None declared.

\section{References}

1 Deuschl G, Toro C, Hallett M. Symptomatic and essential palatal tremor. 2. Differences of palatal movements. Mov Disord 1994;9(6):676-678

2 Choh NA, Choh SA, Jehangir M. Hypertrophic olivary degeneration: the forgotten triangle of Guillain and Mollaret. Neurol India 2009;57(4):507-509

3 Naik KR, Saroja AO. Unilateral symptomatic palatal tremor due to pontocerebellar infarction. Ann Indian Acad Neurol 2011;14(3):219-221 